University of Nebraska - Lincoln

DigitalCommons@University of Nebraska - Lincoln

Papers in the Earth and Atmospheric Sciences

Earth and Atmospheric Sciences, Department

1991

\title{
Dissociation Quotients of Oxalic Acid in Aqueous Sodium Chloride Media to $175 \mathrm{C}$
}

Richard Kettler

University of Nebraska-Lincoln, rkettler1@unl.edu

Donald A. Palmer

Oak Ridge National Laboratory

David J. Wesolowski

Oak Ridge National Laboratory

Follow this and additional works at: https://digitalcommons.unl.edu/geosciencefacpub

Part of the Earth Sciences Commons

Kettler, Richard; Palmer, Donald A.; and Wesolowski, David J., "Dissociation Quotients of Oxalic Acid in Aqueous Sodium Chloride Media to 175C" (1991). Papers in the Earth and Atmospheric Sciences. 195. https://digitalcommons.unl.edu/geosciencefacpub/195

This Article is brought to you for free and open access by the Earth and Atmospheric Sciences, Department of at DigitalCommons@University of Nebraska - Lincoln. It has been accepted for inclusion in Papers in the Earth and Atmospheric Sciences by an authorized administrator of DigitalCommons@University of Nebraska - Lincoln. 


\title{
Dissociation Quotients of Oxalic Acid in Aqueous Sodium Chloride Media to $175^{\circ} \mathrm{C}$
}

\author{
Richard M. Kettler, ${ }^{1}$ Donald A. Palmer, ${ }^{2}$ and \\ David J. Wesolowski ${ }^{2}$ \\ Received January 24, 1991; Revised April 22, 1991
}

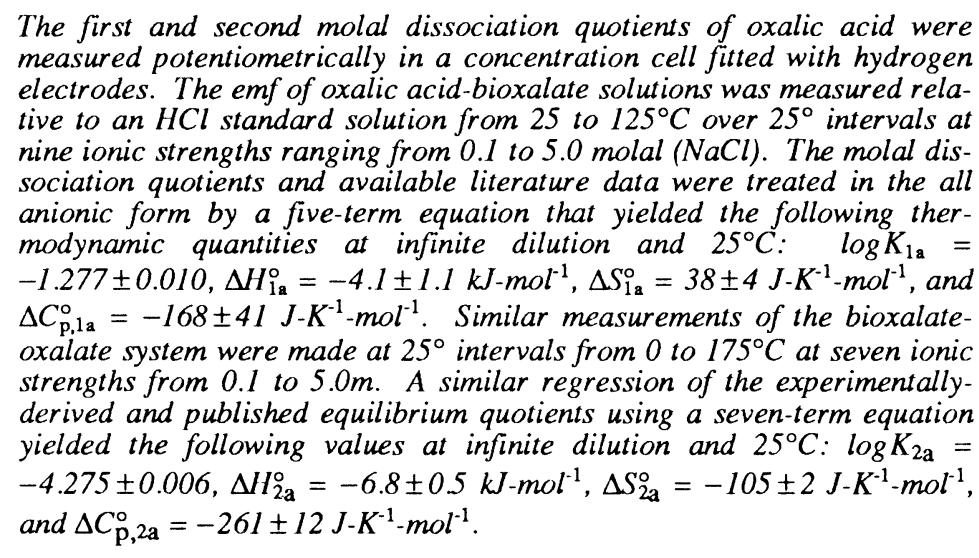

KEY WORDS: Oxalic acid; ethanedioic acid; dissociation constants; thermodynamics; potentiometry; ionic strength.

\section{Introduction}

Knowledge of the dissociation quotients of oxalic (ethanedioic) acid under a wide variety of conditions is important in understanding many processes in materials and geological sciences. Oxalic acid is used in diversified industrial applications, because it provides $\mathrm{pH}$ control and, more importantly, it is capable of chelating ferrous and other metal ions in solution. In particular, oxalate forms stable complexes

\footnotetext{
${ }^{1}$ Department of Geology, University of Nebraska, Lincoln, NE 68588-0340.

${ }^{2}$ Chemistry Division, Oak Ridge National Laboratory, P. O. Box 2008, Building 4500S, Oak Ridge, TN 37831-6110.
} 
with actinides, so that it can be used to decontaminate radioactive components in the cooling systems of reactors. ${ }^{(1)}$ Some actinides form insoluble oxalate salts rendering it useful in the decontamination of radioactive waste. ${ }^{(2)}$ Moreover, there is substantial interest in the corrosion behavior of steels in oxalic acid solutions. ${ }^{(3)}$ Similarly, the importance of oxalic acid to the geological sciences is related to the mobilization of metal ions through complexation. The calcium oxalate minerals whewellite $\left(\mathrm{CaC}_{2} \mathrm{O}_{4} \cdot \mathrm{H}_{2} \mathrm{O}\right)$ and weddellite $\left(\mathrm{CaC}_{2} \mathrm{O}_{4} \cdot 2 \mathrm{H}_{2} \mathrm{O}\right)$ occur in a wide variety of soils and attest to the ubiquity of oxalate species in vadose waters. ${ }^{(4)}$ Recent geological studies have shown that oxalate and bioxalate may act as potential ligands in the dissolution of aluminosilicate minerals during weathering ${ }^{(5)}$ and sedimentary diagenesis. ${ }^{(6)}$ Thus, in any attempts to model these processes quantitatively, the thermodynamic behavior of oxalic acid and the activity/concentration relationships in natural and industrial brines must be considered.

The dissociation of oxalic acid proceeds as follows

$$
\begin{aligned}
& \mathrm{H}_{2} \mathrm{C}_{2} \mathrm{O}_{4} \rightleftarrows \mathrm{H}^{+}+\mathrm{HC}_{2} \mathrm{O}_{4}^{-} \\
& \mathrm{HC}_{2} \mathrm{O}_{4}^{-} \rightleftarrows \mathrm{H}^{+}+\mathrm{C}_{2} \mathrm{O}_{4}^{2-}
\end{aligned}
$$

In spite of the widespread interest in the properties of oxalic acid under hydrothermal conditions, there are relatively few measurements of the dissociation quotients of oxalic acid at elevated temperatures or in solutions of high ionic strength. Although abundant data for equilibria (1) and (2) are available for temperatures between 0 and $50^{\circ} \mathrm{C}$ and for ionic strengths of $\leq 1 \mathrm{~m}$ (Table I), the only data available at elevated temperatures are those of Nikolaeva and Antipina. ${ }^{(17)}$ The latter study was based on measurements that extended to $90^{\circ} \mathrm{C}$ and were made in dilute solutions (ionic strength $<0.13 \mathrm{~m}$ ) in the absence of a supporting electrolyte. In the present research, the first and second dissociation quotients of oxalic acid have been measured potentiometrically at temperatures up to $175^{\circ} \mathrm{C}$ and ionic strengths ranging from 0.1 to $5 \mathrm{~m}$. Knowledge of the magnitude of the first dissociation has little value for most applications because, as can be seen from Table I, oxalic acid is only present to a significant extent in relatively acidic solutions. Moreover, because it is a moderately strong acid, there are the usual experimental difficulties and potentially ambiguous assumptions in the assignment of activity coefficients that arise in determining such constants with precision. ${ }^{(24)}$ 
Table I. Summary of the Thermodynamic Data for the Dissociation of Oxalic Acid

\begin{tabular}{|c|c|c|c|c|c|c|c|c|}
\hline$-\log Q_{1 \mathrm{a}}$ & $\Delta H_{1 \mathrm{a}}{ }^{a}$ & $-\log Q_{2 \mathrm{a}}$ & $\Delta H_{2 \mathrm{a}}{ }^{a}$ & $\Delta C_{\mathrm{p}, 2 \mathrm{a}} b$ & $T\left({ }^{\circ} \mathrm{C}\right)$ & $I(m)$ & Salt & Ref. \\
\hline 1.37 & & 3.81 & & & 25 & 0.10 & $\mathrm{NaClO}_{4}$ & 7 \\
\hline 1.33 & & $3.94^{C}$ & & & 25 & 0.15 & $\mathrm{NaClO}_{4}$ & 8 \\
\hline 1.32 & & 3.63 & & & 5 & 0.50 & $\mathrm{NaClO}_{4}$ & 9 \\
\hline 1.23 & & 3.65 & & & 15 & 0.50 & $\mathrm{NaClO}_{4}$ & 9 \\
\hline 1.20 & & 3.67 & & & 25 & 0.50 & $\mathrm{NaClO}_{4}$ & 9 \\
\hline 1.07 & & 3.57 & & & 25 & 1.00 & $\mathrm{NaClO}_{4}$ & 10 \\
\hline 1.08 & & 3.554 & & & 25 & 1.00 & $\mathrm{NaClO}_{4}$ & 11 \\
\hline 1.01 & & 3.55 & & & 20 & 1.00 & $\mathrm{NaClO}_{4}$ & 12 \\
\hline 0.89 & & 3.51 & & & 25 & 1.00 & $\mathrm{NaCl}$ & 13 \\
\hline \multirow[t]{2}{*}{1.1} & & 3.62 & & & 25 & 1.00 & $\mathrm{KNO}_{3}$ & 14 \\
\hline & $-7.8 \pm 1.5^{d}$ & & $-2.71 \pm 0.02^{d}$ & & 25 & 1.00 & $\mathrm{KNO}_{3}$ & 15 \\
\hline $1.244^{e}$ & & & & & 0 & 0.0 & & 16 \\
\hline $1.252^{e}$ & & & & & 15 & 0.0 & & 16 \\
\hline $1.252^{e}$ & & & & & 25 & 0.0 & & 16 \\
\hline $1.286^{e}$ & & & & & 35 & 0.0 & & 16 \\
\hline $1.295^{e}$ & & & & & 45 & 0.0 & & 16 \\
\hline 1.05 & & 4.26 & & & 25 & 0.0 & & 17 \\
\hline 1.35 & & 4.40 & & & 50 & 0.0 & & 17 \\
\hline 1.54 & & 4.54 & & & 70 & 0.0 & & 17 \\
\hline 1.73 & & 4.71 & & & 90 & 0.0 & & 17 \\
\hline \multirow{12}{*}{\multicolumn{2}{|c|}{$1.299^{e}$}} & 4.300 & & & 25 & 0.0 & & 18 \\
\hline & & $4.228^{e}$ & -1.16 & -180 & 0 & 0.0 & & 19 \\
\hline & & $4.235^{e}$ & -2.10 & -190 & 5 & 0.0 & & 19 \\
\hline & & $4.244^{e}$ & -4.15 & -210 & 10 & 0.0 & & 19 \\
\hline & & $4.255^{e}$ & -5.29 & -220 & 15 & 0.0 & & 19 \\
\hline & & $4.268^{e}$ & -5.29 & -230 & 20 & 0.0 & & 19 \\
\hline & & $4.286^{e}$ & -6.49 & -250 & 25 & 0.0 & & 19 \\
\hline & & $4.308^{e}$ & -7.77 & -260 & 30 & 0.0 & & 19 \\
\hline & & $4.331^{e}$ & -9.11 & -280 & 35 & 0.0 & & 19 \\
\hline & & $4.356^{e}$ & -10.54 & -290 & 40 & 0.0 & & 19 \\
\hline & & $4.388^{e}$ & -12.04 & -310 & 45 & 0.0 & & 19 \\
\hline & & $4.417^{e}$ & -13.62 & -320 & 50 & 0.0 & & 19 \\
\hline \multirow{9}{*}{\multicolumn{2}{|c|}{$1.271^{e}$}} & $4.275^{e}$ & & & 25 & 0.0 & & 20 \\
\hline & & $4.201^{e}$ & -1.447 & -210 & 0 & 0.0 & & 21 \\
\hline & & $4.207^{e}$ & -2.508 & -214 & 5 & 0.0 & & 21 \\
\hline & & $4.218^{e}$ & -3.587 & -218 & 10 & 0.0 & & 21 \\
\hline & & $4.231^{e}$ & -4.686 & -222 & 15 & 0.0 & & 21 \\
\hline & & $4.247^{e}$ & -5.804 & -226 & 20 & 0.0 & & 21 \\
\hline & & $4.266^{e}$ & -6.941 & -229 & 25 & 0.0 & & 21 \\
\hline & & $4.287^{e}$ & -8.098 & -233 & 30 & 0.0 & & 21 \\
\hline & & $4.312^{e}$ & -9.274 & -237 & 35 & 0.0 & & 21 \\
\hline
\end{tabular}


Table I. Continued

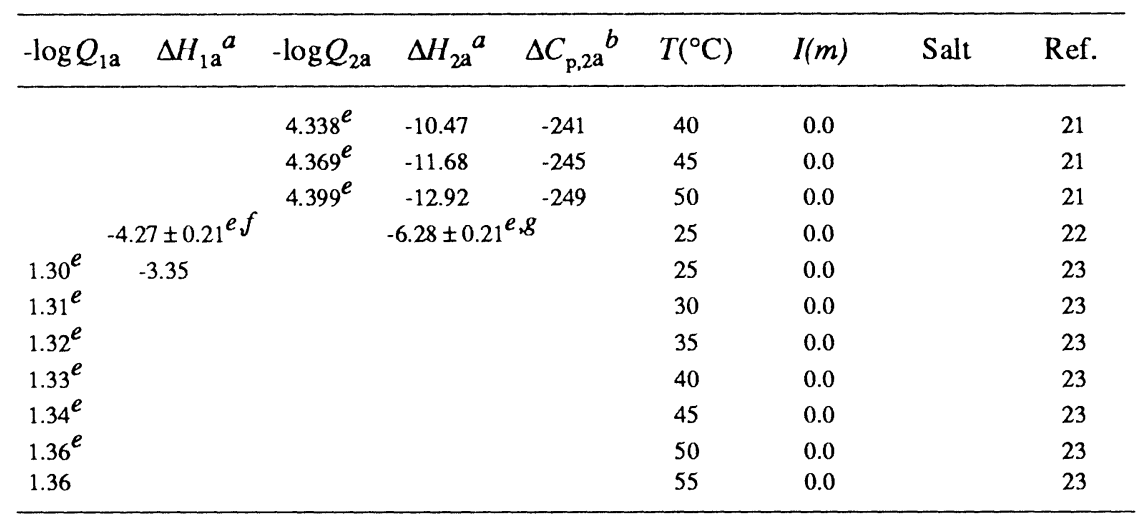

${ }^{a}$ Units: $\mathrm{kJ}^{-\mathrm{mol}^{-1}}{ }^{b}{ }^{b}$ Units: $\mathrm{J}-\mathrm{K}^{-1}-\mathrm{mol}^{-1} .{ }^{c}$ This value was reported incorrectly by A. E. Martell and R. M. Smith, in Critical Stability Constants Complexes, Vol. 5, (First Supplement), (Plenum, New York, 1982), p. 604. ${ }^{d}$ The $\Delta H$ values were based on the apparently erroneous $\log Q$ values recorded by Smith and Martell, viz., 1.04 and 3.55, respectively. ${ }^{e}$ These values were combined with the results of the present study in the fitting process. $f_{\text {The }} \Delta H_{1 \mathrm{a}}^{\circ}$ value is based on the $\log K_{1 \mathrm{a}}$ reported in Ref. $20 .{ }^{g}$ The $\Delta \mathrm{H}_{2 \mathrm{a}}$ value is based on the $\log K_{2 \mathrm{a}}$ reported in Ref. 21 .

\section{Experimental}

\subsection{Materials}

Stock solutions of $\mathrm{NaCl}, \mathrm{NaOH}$ and $\mathrm{HCl}$ were prepared from reagent grade chemicals, sparged with argon and stored under argon in polypropylene containers. Water was purified by passing distilled water through a Barnstead four stage deionizing system that yielded water with a resistivity of $0.18 \mathrm{M} \Omega-\mathrm{m}$. All solutions were made by combining reagent grade $\mathrm{H}_{2} \mathrm{C}_{2} \mathrm{O}_{4} \cdot 2 \mathrm{H}_{2} \mathrm{O}$ with the $\mathrm{NaOH}, \mathrm{HCl}$ and $\mathrm{NaCl}$ stock solutions and water. Compositions of the solutions used for experimental runs are given in Table II. Two stock reference cell solutions with ionic strengths of 2.0 and 5.0 molal were prepared (ionic strength was determined from stoichiometric molalities of all ions in solution) and diluted to the appropriate ionic strength prior to each experiment. The molal ratio of $\mathrm{HCl}$ to $\mathrm{NaCl}$ in these solutions was $1 / 19$ in the 2 molal solution and $1 / 500$ in the 5 molal solution. Solutions used to determine the first dissociation quotient contained variable proportions of oxalic acid, $\mathrm{NaCl}$, and $\mathrm{HCl}$. The ratio of total oxalate to $\mathrm{NaCl}$ was less than $1 / 100$ in all runs except for those at ionic strengths of 0.1 and 0.3 (Table II). In 
Table II. Starting Molal Solution Compositions

\begin{tabular}{|c|c|c|c|c|c|c|}
\hline \multirow[b]{2}{*}{$\begin{array}{l}\text { Run } \\
\text { No. }\end{array}$} & \multirow[b]{2}{*}{$\underset{(\mathrm{NaCl})}{m}$} & \multicolumn{2}{|c|}{ Test Cell } & \multirow[b]{2}{*}{$\begin{array}{c}m \\
(\mathrm{HCl})\end{array}$} & \multicolumn{2}{|c|}{ Ref. Cell } \\
\hline & & $\stackrel{m}{\left.\stackrel{m}{\mathrm{H}_{2}} \mathrm{O}_{4}\right)}$ & $\begin{array}{c}m \\
(\mathrm{NaOH})\end{array}$ & & $\begin{array}{c}m \\
(\mathrm{NaCl})\end{array}$ & $\begin{array}{c}m \\
(\mathrm{HCl})\end{array}$ \\
\hline 1,2 & 1.991 & 0.02003 & 0.03003 & 0.0 & 1.900 & 0.1039 \\
\hline 3 & 0.9807 & 0.01002 & 0.01502 & 0.0 & 0.9501 & 0.05200 \\
\hline 4 & 0.9806 & 0.01002 & 0.01502 & 0.0 & 0.9501 & 0.05200 \\
\hline 5 & 0.3269 & 0.003340 & 0.005006 & 0.0 & 0.3167 & 0.01732 \\
\hline 6 & 0.09808 & 0.001002 & 0.001502 & 0.0 & 0.09501 & 0.005197 \\
\hline 7 & 0.3268 & 0.003339 & 0.005004 & 0.0 & 0.3167 & 0.01732 \\
\hline 8 & 1.961 & 0.02003 & 0.03003 & 0.0 & 1.900 & 0.1039 \\
\hline 9 & 0.9803 & 0.01002 & 0.01501 & 0.0 & 0.9498 & 0.05196 \\
\hline 10 & 0.9804 & 0.01002 & 0.01501 & 0.0 & 0.9499 & 0.05196 \\
\hline $11,12,14$ & 4.980 & 0.01003 & 0.01500 & 0.0 & 4.990 & 0.009999 \\
\hline 13,15 & 3.136 & 0.006313 & 0.009447 & 0.0 & 3.141 & 0.006295 \\
\hline 16 & 0.1813 & 0.001853 & 0.002777 & 0.0 & 0.1754 & 0.009596 \\
\hline 17,18 & 2.000 & 0.01039 & 0.0 & 0.0 & 1.900 & 0.1039 \\
\hline 19 & 4.965 & 0.02114 & 0.0 & 0.01999 & 4.990 & 0.009999 \\
\hline 20 & 1.965 & 0.01999 & 0.0 & 0.01999 & 1.900 & 0.1039 \\
\hline 21 & 0.08494 & 0.01985 & 0.0 & 0.0 & 0.09499 & 0.005196 \\
\hline 22,26 & 3.123 & 0.01330 & 0.0 & 0.04003 & 3.157 & 0.006326 \\
\hline 23 & 0.9826 & 0.009998 & 0.0 & 0.04042 & 0.9764 & 0.05308 \\
\hline 24 & 0.3150 & 0.01983 & 0.0 & 0.0 & 0.3127 & 0.01710 \\
\hline 25 & 1.927 & 0.01961 & 0.0 & 0.03998 & 1.875 & 0.1026 \\
\hline 27 & 3.973 & 0.01691 & 0.0 & 0.01600 & 3.991 & 0.007997 \\
\hline 28 & 2.979 & 0.01268 & 0.0 & 0.01200 & 2.994 & 0.006000 \\
\hline 29,30 & 0.2949 & 0.003000 & 0.0 & 0.003001 & 0.2994 & 0.003040 \\
\hline 31 & 0.2751 & 0.02015 & 0.0 & 0.02000 & 0.2750 & 0.02500 \\
\hline
\end{tabular}

these more dilute solutions, the ratio of oxalate to supporting electrolyte was as large as 1/4.3 (Table II). Two stock solutions with ionic strengths of 2.0 and 5.0 molal were prepared for those experiments aimed at determining the second dissociation constant. The molal ratio of $\mathrm{H}_{2} \mathrm{C}_{2} \mathrm{O}_{4}$ to $\mathrm{NaOH}$ was $\mathrm{ca}$. $2 / 3$ in both solutions with a molal ratio of total oxalate to supporting electrolyte of $1 / 100$ in the 2 molal solution and $1 / 2000$ in the 5 molal solution. These solutions were diluted to the appropriate ionic strengths prior to each experiment. Ultrapure Matheson, $99.999 \%$, hydrogen was used to saturate the cell solutions. 


\subsection{Apparatus and Procedures}

The concentration cell used in this work was described by Mesmer et al. ${ }^{(25)}$ with some modifications described by Wesolowski $e t$ $a l .{ }^{(26)}$ More recently, the oil bath was replaced by an Isotherm 3002 model (Hart Scientific) with an improved stability of $\pm 0.02^{\circ} \mathrm{C}$ over the duration of an experiment. A permanent magnet, that is rotated via a chain-drive mechanism, is now immersed in the bath enabling the solutions within the pressure vessel to be stirred efficiently. Moreover in this study, the emf, temperature, and pressure readings were monitored with a microcomputer and the averages of 100 readings were recorded every four minutes to improve the signal to noise ratio. Prior to each experiment, the porous Teflon liquid junction was repacked and saturated with a $5.4 \mathrm{~m} \mathrm{NaCl}$ solution, and the platinum electrodes were coated with fresh platinum black.

In the experiments designed to measure the second dissociation quotient (experiments 1-16, Table II), the cell configuration was

$$
\mathrm{Pt}, \mathrm{H}_{2}\left|\mathrm{NaHC}_{2} \mathrm{O}_{4}, \mathrm{NaCl}, \mathrm{NaOH} \| \mathrm{NaCl}, \mathrm{HCl}\right| \mathrm{H}_{2}, \mathrm{Pt}
$$

Experiments 17-31 (Table II) were designed to measure the first dissociation quotient using the cell configuration

$$
\mathrm{Pt}, \mathrm{H}_{2}\left|\mathrm{H}_{2} \mathrm{C}_{2} \mathrm{O}_{4}, \mathrm{NaCl}, \mathrm{HCl} \| \mathrm{NaCl}, \mathrm{HCl}\right| \mathrm{H}_{2}, \mathrm{Pt}
$$

In order to initiate an experiment, the test and reference stock solutions were diluted to equal ionic strengths and placed in the concentration cell. The cell was then purged five times with hydrogen by pressurizing the vessel to approximately $3.3 \mathrm{MPa}$ and venting to ambient pressure. After the purging process was completed, the hydrogen pressure was regulated to approximately $3.3 \mathrm{MPa}$ and the vessel was sealed and immersed in a thermostatted bath (temperature stability was $\pm 0.02^{\circ} \mathrm{C}$ for the oil bath, which was used at $\geq 75^{\circ} \mathrm{C}$, and $\pm 0.05^{\circ} \mathrm{C}$ for the water/ice bath, used in the $0-75^{\circ} \mathrm{C}$ runs) with continuous stirring. After the cell reached thermal equilibrium at $25^{\circ} \mathrm{C}$ and a stable potential was achieved (usually overnight), the potential, pressure, and temperature were recorded. The temperature was then lowered to $0^{\circ} \mathrm{C}$, then raised to $50^{\circ} \mathrm{C}$ and, thereafter, increased in $25^{\circ} \mathrm{C}$ increments, with data recorded at each temperature.

In the oxalate/bioxalate experiments at temperatures of $150-175^{\circ} \mathrm{C}$, drifting potentials (to higher absolute values) were observed that were attributable to thermal decomposition of oxalate. A linear cor- 
rection to the drifting potentials was applied by back extrapolation to the time at which the vessel reached thermal equilibrium. The drift corrections were reproducible and were generally less than $0.02 \mathrm{mV}-\mathrm{min}^{-1}$ at $150^{\circ} \mathrm{C}$ and less than $0.16 \mathrm{mV}-\mathrm{min}^{-1}$ at $175^{\circ} \mathrm{C}$.

Apparent decomposition was observed at even lower temperatures in the more acidic solutions used to determine the first dissociation quotient, which is consistent with the acid-catalyzed rate of decarboxylation observed by Crossey. ${ }^{(27)}$ In these experiments, stable potentials were observed through $125^{\circ} \mathrm{C}$, while the drift at $150^{\circ} \mathrm{C}$ was sufficiently rapid that reliable results could not be obtained.

\section{Results}

The observed potential is related to the cell concentrations by the relationship

$$
E=(R T / F) \ln \left(\left[\mathrm{H}^{+}\right]_{\mathrm{t}} /\left[\mathrm{H}^{+}\right]_{\mathrm{r}}\right)-\sum_{i} D_{\mathrm{i}}\left([i]_{\mathrm{r}}-[i]_{\mathrm{t}}\right)
$$

where the square brackets designate the molality of the enclosed ionic species and the subscripts $t$ and $r$ refer to the test and reference solutions, respectively. Absolute temperature is denoted by $T$, and the ideal gas and Faraday constants are designated by $R$ and $F$, respectively. Molal concentrations, rather than activities, can be used because the ratio of activity coefficients is assumed to be unity in the matching ionic media of the test and reference compartments (Table II). The second term approximates the liquid junction potential as calculated from the Henderson equation in a form simplified by the presence of similar ionic media in each half cell. ${ }^{(28)}$ Limiting equivalent conductances (required to calculate $D_{\mathrm{i}}$ ) for all the ionic species are taken from Quist and Marshall. (29) Limiting equivalent conductances for oxalate and bioxalate are not available at high temperatures and were assumed, therefore, to be equal to those for sulfate and bisulfate, respectively. This approach follows previous reports from this laboratory. ${ }^{(26,30)}$ The molal ratio of $\mathrm{NaCl}$ to total oxalate is greater than 100 with the exception of experiments 21, 24 and 31 (Table II); thus, the presence of the oxalate species will contribute relatively little to the liquid junction potential.

The respective molal stoichiometric reaction quoticnts for Reactions 1 and 2 are defined as

$$
\begin{aligned}
& Q_{1 \mathrm{a}}=\left[\mathrm{H}^{+}\right]\left[\mathrm{HC}_{2} \mathrm{O}_{4}^{-}\right] /\left[\mathrm{H}_{2} \mathrm{C}_{2} \mathrm{O}_{4}\right] \\
& Q_{2 \mathrm{a}}=\left[\mathrm{H}^{+}\right]\left[\mathrm{C}_{2} \mathrm{O}_{4}^{2-}\right] /\left[\mathrm{HC}_{2} \mathrm{O}_{4}^{-}\right]
\end{aligned}
$$


The reaction quotients are related to the observed potential by

$$
\begin{aligned}
\log Q_{\mathrm{xa}}= & \log \left[\mathrm{H}^{+}\right]_{\mathrm{r}}+F /(2.3026 R T)\left\{E+\sum_{i} D_{\mathrm{i}}\left([i]_{\mathrm{r}}-[i]_{\mathrm{t}}\right)\right\} \\
& +\log \left(\left[\mathrm{H}_{2-\mathrm{x}} \mathrm{C}_{2} \mathrm{O}_{4}^{-\mathrm{x}}\right] /\left[\mathrm{H}_{3-\mathrm{x}} \mathrm{C}_{2} \mathrm{O}_{4}^{1-\mathrm{x}}\right]\right)
\end{aligned}
$$

where $x=1$ or 2 . The exact concentrations of the oxalate species are computed from the known total concentration of oxalate, the reference hydrogen ion molality, and the cell potential using an iterative calculation that involves oxalate speciation, ionic strength, and liquid junction potential. ${ }^{(26,30-32)}$ The results of this calculation are given in Tables III and IV which list the $\mathrm{pH}$ (defined here as the negative logarithm of the hydrogen ion molality), ionic strength (considering the actual distribution of ionic species), the degree of association of oxalate $(\bar{n})$, and log $Q_{\mathrm{x}}$. For the purpose of these calculations, the degree of association is defined by

$$
\bar{n}=\left\{\left[\mathrm{H}^{+}\right]-\left[\mathrm{OH}^{-}\right]-m(\mathrm{HCl})\right\} / m\left(\mathrm{H}_{2} \mathrm{C}_{2} \mathrm{O}_{4}\right)
$$

where $m(\mathrm{HCl})$ and $m\left(\mathrm{H}_{2} \mathrm{C}_{2} \mathrm{O}_{4}\right)$ are the total molal concentrations computed from the starting solution concentrations (Table II), ignoring dissociation of oxalic acid and bioxalate. The values of uncertainties in $\bar{n}$, $\sigma$, and hence those in $\log Q_{\mathrm{x}}, \sigma_{\mathrm{x}}$, were computed from the sum of 18 estimated experimental and computational errors, including those derived from the liquid junction potential calculation.

\section{Discussion}

Combination of the equation for the dissociation of water with reactions 1 and 2 , leads to the following equilibria in their respective base, or anionic, forms

$$
\begin{aligned}
& \mathrm{H}_{2} \mathrm{C}_{2} \mathrm{O}_{4}+\mathrm{OH}^{-} \rightleftarrows \mathrm{HC}_{2} \mathrm{O}_{4}^{-}+\mathrm{H}_{2} \mathrm{O} \\
& \mathrm{HC}_{2} \mathrm{O}_{4}^{-}+\mathrm{OH}^{-} \rightleftarrows \mathrm{C}_{2} \mathrm{O}_{4}^{2-}+\mathrm{H}_{2} \mathrm{O}
\end{aligned}
$$

The equilibrium quotients for these reactions are written as

$$
\begin{aligned}
Q_{1 \mathrm{~b}} & =\left[\mathrm{HC}_{2} \mathrm{O}_{4}^{-}\right] /\left[\mathrm{H}_{2} \mathrm{C}_{2} \mathrm{O}_{4}\right]\left[\mathrm{OH}^{-}\right] \\
Q_{2 \mathrm{~b}} & =\left[\mathrm{C}_{2} \mathrm{O}_{4}^{2-}\right] /\left[\mathrm{HC}_{2} \mathrm{O}_{4}^{-}\right]\left[\mathrm{OH}^{-}\right]
\end{aligned}
$$

The values of $Q_{1 \mathrm{a}}$ and $\mathrm{Q}_{2 \mathrm{a}}$ (Tables III and IV) were converted to the corresponding base forms (Tables III and IV) using the equilibrium 
Table III. Experimental Results for the First Dissociation Quotient of Oxalic Acid

\begin{tabular}{|c|c|c|c|c|c|c|c|c|c|c|c|}
\hline Expt. & $\begin{array}{c}T \\
\left({ }^{\circ} \mathrm{C}\right)\end{array}$ & $\begin{array}{c}I \\
(m)\end{array}$ & $\begin{array}{c}\mathrm{emf} \\
(\mathrm{mV})\end{array}$ & $\begin{array}{c}\text { elj } \\
(\mathrm{mV})\end{array}$ & $\mathrm{pH}$ & $\bar{n}$ & $\sigma$ & $\mathrm{p} Q_{1 \mathrm{a}}$ & $\sigma_{1 \mathrm{a}}$ & $-\mathrm{p} Q_{\mathrm{lb}}$ & $\sigma_{1 b}$ \\
\hline \multirow[t]{4}{*}{21} & 49.85 & 0.101 & 25.88 & -5.70 & 1.792 & 1.199 & 0.024 & 1.172 & 0.067 & 11.866 & 0.073 \\
\hline & 74.83 & 0.101 & 27.90 & -5.18 & 1.805 & 1.22 & 0.019 & 1.247 & 0.049 & 11.213 & 0.055 \\
\hline & 99.79 & 0.100 & 29.49 & -4.64 & 1.823 & 1.249 & 0.015 & 1.338 & 0.034 & 10.658 & 0.040 \\
\hline & 124.77 & 0.099 & 30.54 & -4.19 & 1.844 & 1.844 & 0.012 & 1.438 & 0.025 & 10.187 & 0.031 \\
\hline \multirow[t]{4}{*}{24} & 49.89 & 0.332 & -0.23 & -0.23 & 1.767 & 1.157 & 0.008 & 1.010 & 0.025 & 11.954 & 0.034 \\
\hline & 74.82 & 0.332 & -0.94 & -0.19 & 1.778 & 1.173 & 0.007 & 1.081 & 0.022 & 11.296 & 0.031 \\
\hline & 99.79 & 0.331 & -2.02 & -0.16 & 1.792 & 1.197 & 0.007 & 1.169 & 0.019 & 10.732 & 0.028 \\
\hline & 124.77 & 0.331 & -3.52 & -0.09 & 1.810 & 1.227 & 0.007 & 1.271 & 0.017 & 10.243 & 0.026 \\
\hline \multirow[t]{4}{*}{23} & 49.85 & 1.030 & -2.74 & 0.22 & 1.321 & 1.278 & 0.020 & 0.901 & 0.044 & 12.064 & 0.054 \\
\hline & 74.82 & 1.030 & -3.14 & 0.21 & 1.323 & 1.301 & 0.019 & 0.952 & 0.039 & 11.411 & 0.049 \\
\hline & 99.79 & 1.030 & -3.67 & 0.19 & 1.327 & 1.340 & 0.017 & 1.035 & 0.034 & 10.833 & 0.044 \\
\hline & 124.77 & 1.030 & -4.34 & 0.20 & 1.332 & 1.395 & 0.016 & 1.145 & 0.029 & 10.311 & 0.039 \\
\hline \multirow[t]{4}{*}{25} & 49.86 & 1.982 & -16.42 & 1.21 & 1.264 & 1.271 & 0.019 & 0.827 & 0.042 & 12.234 & 0.052 \\
\hline & 74.82 & 1.982 & -18.08 & 1.09 & 1.267 & 1.286 & 0.017 & 0.864 & 0.036 & 11.581 & 0.046 \\
\hline & 99.79 & 1.982 & -19.90 & 0.98 & 1.271 & 1.313 & 0.015 & 0.925 & 0.030 & 11.007 & 0.040 \\
\hline & 124.77 & 1.982 & -21.97 & 0.93 & 1.279 & 1.361 & 0.014 & 1.028 & 0.026 & 10.469 & 0.036 \\
\hline \multirow[t]{5}{*}{20} & 24.86 & 2.001 & -25.83 & 1.88 & 1.452 & 1.248 & 0.018 & 0.957 & 0.041 & 12.863 & 0.057 \\
\hline & 49.83 & 2.002 & -27.58 & 1.68 & 1.440 & 1.197 & 0.016 & 0.815 & 0.043 & 12.249 & 0.059 \\
\hline & 74.82 & 2.002 & -30.23 & 1.51 & 1.443 & 1.208 & 0.014 & 0.851 & 0.036 & 11.598 & 0.052 \\
\hline & 99.79 & 2.002 & -33.06 & 1.34 & 1.448 & 1.227 & 0.012 & 0.908 & 0.030 & 11.026 & 0.046 \\
\hline & 124.77 & 2.002 & -36.16 & 1.27 & 1.457 & 1.262 & 0.011 & 1.001 & 0.024 & 10.498 & 0.041 \\
\hline \multirow[t]{5}{*}{28} & 24.86 & 3.002 & 33.69 & -0.35 & 1.646 & 1.188 & 0.008 & 0.983 & 0.022 & 12.979 & 0.038 \\
\hline & 49.93 & 3.002 & 36.60 & -0.32 & 1.646 & 1.186 & 0.007 & 0.979 & 0.021 & 12.218 & 0.037 \\
\hline & 74.84 & 3.003 & 39.25 & -0.28 & 1.649 & 1.197 & 0.007 & 1.018 & 0.018 & 11.554 & 0.034 \\
\hline & 99.80 & 3.004 & 41.81 & -0.25 & 1.653 & 1.212 & 0.006 & 1.064 & 0.016 & 10.978 & 0.032 \\
\hline & 124.78 & 3.006 & 44.27 & -0.23 & 1.658 & 1.228 & 0.006 & 1.114 & 0.014 & 10.471 & 0.030 \\
\hline \multirow[t]{4}{*}{22} & 49.84 & 3.172 & 56.23 & -0.72 & 1.310 & 1.337 & 0.019 & 1.011 & 0.037 & 12.213 & 0.053 \\
\hline & 74.76 & 3.173 & 60.44 & -0.64 & 1.368 & 1.368 & 0.017 & 1.075 & 0.031 & 11.521 & 0.047 \\
\hline & 99.79 & 3.173 & 64.69 & -0.56 & 1.392 & 1.392 & 0.015 & 1.124 & 0.028 & 10.938 & 0.044 \\
\hline & 124.77 & 3.175 & 68.78 & -0.52 & 1.426 & 1.426 & 0.014 & 1.188 & 0.025 & 10.413 & 0.041 \\
\hline \multirow[t]{5}{*}{27} & 24.92 & 4.002 & 32.77 & -0.33 & 1.537 & 1.244 & 0.008 & 1.034 & 0.018 & 13.084 & 0.048 \\
\hline & 49.89 & 4.002 & 35.57 & -0.30 & 1.537 & 1.245 & 0.007 & 1.035 & 0.016 & 12.316 & 0.046 \\
\hline & 74.86 & 4.003 & 38.25 & -0.27 & 1.539 & 1.251 & 0.006 & 1.052 & 0.015 & 11.661 & 0.045 \\
\hline & 99.81 & 4.004 & 40.67 & -0.24 & 1.544 & 1.269 & 0.006 & 1.100 & 0.013 & 11.067 & 0.043 \\
\hline & 124.77 & 4.007 & 42.69 & -0.22 & 1.553 & 1.304 & 0.005 & 1.186 & 0.011 & 10.505 & 0.041 \\
\hline \multirow[t]{4}{*}{19} & 24.88 & 4.999 & 31.00 & -0.30 & 1.471 & 1.353 & 0.007 & 1.203 & 0.013 & 13.085 & 0.043 \\
\hline & 49.85 & 5.000 & 34.18 & -0.20 & 1.462 & 1.323 & 0.007 & 1.135 & 0.013 & 12.381 & 0.043 \\
\hline & 74.81 & 5.001 & 36.93 & -0.26 & 1.461 & 1.320 & 0.006 & 1.127 & 0.012 & 11.741 & 0.042 \\
\hline & 99.79 & 5.001 & 39.49 & -0.23 & 1.463 & 1.326 & 0.006 & 1.143 & 0.011 & 11.162 & 0.041 \\
\hline
\end{tabular}

quotients for the dissociation of water in $\mathrm{NaCl}$ media obtained by Busey and Mesmer. ${ }^{(33)}$ The base forms of the dissociation reactions are isocoulombic, at least in terms of total anionic charge. Therefore, the equilibrium quotients are expected to have a much simpler dependence on ionic strength (Figs. 1 and 2), as is particularly noticeable for reac- 
Table IV. Experimental Results for the Second Dissociation Quotient of Oxalic Acid

\begin{tabular}{|c|c|c|c|c|c|c|c|c|c|c|c|}
\hline Expt. & $\begin{array}{c}T \\
\left({ }^{\circ} \mathrm{C}\right)\end{array}$ & $\begin{array}{c}I \\
(m)\end{array}$ & $\begin{array}{c}\mathrm{emf} \\
(\mathrm{mV})\end{array}$ & $\begin{array}{c}\text { elj } \\
(\mathrm{mV})\end{array}$ & $\mathrm{pH}$ & $\bar{n}$ & $\sigma$ & $\mathrm{p} Q_{2 \mathrm{a}}$ & $\sigma_{2 \mathrm{a}}$ & $-\mathrm{p} Q_{2 \mathrm{~b}}$ & $\sigma_{2 b}$ \\
\hline \multirow[t]{7}{*}{6} & 24.92 & 0.100 & -97.76 & 2.80 & 3.985 & 0.396 & 0.007 & 3.801 & 0.012 & 9.971 & 0.018 \\
\hline & 49.84 & 0.100 & -111.35 & 2.53 & 4.061 & 0.413 & 0.006 & 3.909 & 0.012 & 9.132 & 0.018 \\
\hline & 74.77 & 0.100 & -127.88 & 2.26 & 4.169 & 0.432 & 0.006 & 4.052 & 0.011 & 8.412 & 0.017 \\
\hline & 99.79 & 0.100 & -147.46 & 2.00 & 4.304 & 0.451 & 0.006 & 4.218 & 0.011 & 7.781 & 0.017 \\
\hline & 124.77 & 0.100 & -170.27 & 1.86 & 4.405 & 0.466 & 0.006 & 4.405 & 0.011 & 7.221 & 0.017 \\
\hline & 149.76 & 0.100 & -196.25 & 1.72 & 4.604 & 0.478 & 0.006 & 4.604 & 0.011 & 6.725 & 0.017 \\
\hline & 174.78 & 0.100 & -227.30 & 1.58 & 4.858 & 0.487 & 0.006 & 4.836 & 0.011 & 6.262 & 0.017 \\
\hline \multirow[t]{8}{*}{16} & 0.00 & 0.185 & -93.55 & 3.17 & 3.803 & 0.412 & 0.007 & 3.650 & 0.012 & 11.040 & 0.018 \\
\hline & 24.93 & 0.185 & -104.95 & 2.82 & 3.840 & 0.421 & 0.007 & 3.650 & 0.012 & 10.031 & 0.018 \\
\hline & 49.87 & 0.185 & -118.94 & 2.54 & 3.913 & 0.434 & 0.006 & 3.798 & 0.011 & 9.200 & 0.017 \\
\hline & 74.86 & 0.185 & -136.41 & 2.28 & 4.026 & 0.449 & 0.006 & 3.938 & 0.011 & 8.477 & 0.017 \\
\hline & 99.77 & 0.185 & -157.42 & 2.01 & 4.172 & 0.464 & 0.006 & 4.110 & 0.011 & 7.837 & 0.017 \\
\hline & 124.77 & 0.185 & -180.86 & 1.87 & 4.332 & 0.475 & 0.006 & 4.289 & 0.011 & 7.278 & 0.017 \\
\hline & 149.77 & 0.185 & -208.22 & 1.73 & 4.519 & 0.484 & 0.006 & 4.492 & 0.011 & 6.771 & 0.017 \\
\hline & 174.72 & 0.186 & -241.66 & 1.58 & 4.754 & 0.491 & 0.006 & 4.739 & 0.011 & 6.285 & 0.017 \\
\hline \multirow[t]{7}{*}{5} & 24.88 & 0.334 & -112.66 & 2.82 & 3.714 & 0.442 & 0.006 & 3.612 & 0.011 & 10.094 & 0.020 \\
\hline & 49.88 & 0.334 & -127.52 & 2.55 & 3.791 & 0.451 & 0.006 & 3.706 & 0.011 & 9.259 & 0.020 \\
\hline & 74.84 & 0.334 & -145.24 & 2.28 & 3.898 & 0.462 & 0.006 & 3.832 & 0.011 & 8.545 & 0.020 \\
\hline & 99.79 & 0.334 & -165.79 & 2.01 & 4.029 & 0.472 & 0.006 & 3.980 & 0.011 & 7.921 & 0.020 \\
\hline & 124.77 & 0.334 & -189.04 & 1.87 & 4.179 & 0.480 & 0.006 & 4.144 & 0.011 & 7.369 & 0.020 \\
\hline & 149.76 & 0.334 & -215.11 & 1.72 & 4.345 & 0.487 & 0.006 & 4.322 & 0.011 & 6.879 & 0.020 \\
\hline & 174.77 & 0.335 & -242.80 & 1.58 & 4.510 & 0.491 & 0.006 & 4.494 & 0.011 & 6.456 & 0.020 \\
\hline \multirow[t]{2}{*}{7} & 0.00 & 0.334 & -101.08 & 3.19 & 3.685 & 0.435 & 0.006 & 3.573 & 0.011 & 11.092 & 0.020 \\
\hline & 24.91 & 0.334 & -112.92 & 2.82 & 3.719 & 0.441 & 0.006 & 3.618 & 0.011 & 10.090 & 0.020 \\
\hline \multirow[t]{4}{*}{3} & 99.80 & 1.001 & -183.77 & 2.01 & 3.795 & 0.484 & 0.006 & 3.767 & 0.011 & 8.099 & 0.021 \\
\hline & 124.77 & 1.002 & -206.18 & 1.87 & 3.919 & 0.488 & 0.006 & 3.898 & 0.011 & 7.557 & 0.021 \\
\hline & 149.77 & 1.003 & -230.59 & 1.73 & 4.052 & 0.491 & 0.006 & 4.036 & 0.011 & 7.077 & 0.021 \\
\hline & 174.77 & 1.005 & -260.00 & 1.58 & 4.226 & 0.494 & 0.006 & 4.216 & 0.011 & 6.616 & 0.021 \\
\hline \multirow[t]{3}{*}{4} & 24.90 & 1.001 & -133.32 & 2.85 & 3.587 & 0.473 & 0.006 & $3.54 \mathrm{i}$ & 0.011 & 10.170 & 0.021 \\
\hline & 49.87 & 1.001 & -146.15 & 2.56 & 3.605 & 0.474 & 0.006 & 3.561 & 0.011 & 9.401 & 0.021 \\
\hline & 74.80 & 1.001 & -163.49 & 2.29 & 3.685 & 0.479 & 0.006 & 3.649 & 0.011 & 8.712 & 0.021 \\
\hline 9 & 24.90 & 1.001 & -133.93 & 2.85 & 3.597 & 0.474 & 0.606 & 3.556 & 0.011 & 10.155 & 0.021 \\
\hline 10 & 24.90 & 1.001 & -134.11 & 2.85 & 3.600 & 0.474 & 0.006 & 3.552 & 0.011 & 10.159 & 0.021 \\
\hline \multirow[t]{4}{*}{1} & 99.79 & 2.002 & -198.77 & 2.02 & 3.696 & $0.4 \subseteq 0$ & 0.006 & 3.679 & 0.011 & 8.254 & 0.027 \\
\hline & 124.79 & 2.004 & -219.91 & 1.87 & 3.792 & 0.491 & 0.006 & 3.777 & 0.011 & 7.719 & 0.027 \\
\hline & 149.76 & 2.006 & -242.40 & 1.73 & 3.892 & 0.493 & 0.006 & 3.880 & 0.011 & 7.246 & 0.027 \\
\hline & 174.81 & 2.009 & -265.490 & 1.58 & 3.986 & 0.494 & 0.006 & 3.977 & 0.011 & 6.832 & 0.027 \\
\hline \multirow[t]{4}{*}{2} & 24.93 & 2.002 & -145.74 & 2.85 & 3.496 & 0.482 & 0.006 & 3.466 & 0.011 & 10.351 & 0.027 \\
\hline & 49.90 & 2.002 & -157.91 & 2.57 & 3.514 & 0.484 & 0.006 & 3.486 & 0.011 & 9.576 & 0.027 \\
\hline & 74.81 & 2.002 & -177.78 & 2.29 & 3.591 & 0.487 & 0.006 & 3.569 & 0.011 & 8.879 & 0.027 \\
\hline & 99.81 & 2.002 & -198.57 & 2.02 & 3.694 & 0.489 & 0.006 & 3.676 & 0.011 & 8.258 & 0.027 \\
\hline \multirow[t]{3}{*}{8} & 0.00 & 2.002 & -137.94 & 3.22 & 3.532 & 0.482 & 0.006 & 3.503 & 0.011 & 11.281 & 0.027 \\
\hline & 24.90 & 2.002 & -147.32 & 2.85 & 3.523 & 0.484 & 0.006 & 3.495 & 0.011 & 10.327 & 0.027 \\
\hline & 49.89 & 2.002 & -162.59 & 2.57 & 3.560 & 0.485 & 0.006 & 3.535 & 0.011 & 9.530 & 0.027 \\
\hline \multirow[t]{6}{*}{13} & 49.89 & 3.149 & -89.16 & 0.08 & 3.593 & 0.461 & 0.006 & 3.526 & 0.011 & 9.694 & 0.027 \\
\hline & 74.82 & 3.149 & -98.18 & 0.07 & 3.624 & 0.464 & 0.006 & 3.562 & 0.011 & 9.031 & 0.027 \\
\hline & 99.83 & 3.151 & -108.83 & 0.06 & 3.672 & 0.468 & 0.006 & 3.617 & 0.011 & 8.442 & 0.027 \\
\hline & 124.83 & 3.153 & -120.77 & 0.05 & 3.730 & 0.472 & 0.006 & 3.683 & 0.011 & 7.915 & 0.027 \\
\hline & 149.76 & 3.156 & -134.39 & 0.04 & 3.802 & 0.476 & 0.006 & 3.762 & 0.011 & 7.436 & 0.027 \\
\hline & 174.78 & 3.161 & -149.50 & 0.04 & 3.882 & 0.481 & 0.006 & 3.849 & 0.011 & 6.998 & 0.027 \\
\hline
\end{tabular}


Table IV. Continued

\begin{tabular}{|c|c|c|c|c|c|c|c|c|c|c|c|}
\hline Expt. & $\begin{array}{c}T \\
\left({ }^{\circ} \mathrm{C}\right)\end{array}$ & $\begin{array}{c}I \\
(m)\end{array}$ & $\begin{array}{l}\text { emf } \\
(\mathrm{mV})\end{array}$ & $\begin{array}{c}\mathrm{elj} \\
(\mathrm{mV})\end{array}$ & $\mathrm{pH}$ & $\bar{n}$ & $\sigma$ & $\mathrm{p} Q_{2 \mathrm{a}}$ & $\sigma_{2 \mathrm{a}}$ & $-\mathrm{p} Q_{2 \mathrm{~b}}$ & $\sigma_{2 b}$ \\
\hline \multirow[t]{4}{*}{15} & 0.00 & 3.150 & -76.60 & 0.11 & 3.616 & 0.461 & 0.006 & 3.550 & 0.011 & 11.391 & 0.027 \\
\hline & 24.92 & 3.150 & -81.80 & 0.09 & 3.586 & 0.460 & 0.006 & 3.517 & 0.011 & 10.465 & 0.027 \\
\hline & 49.87 & 3.150 & -89.32 & 0.08 & 3.596 & 0.461 & 0.006 & 3.529 & 0.011 & 9.691 & 0.027 \\
\hline & 74.83 & 3.150 & -98.88 & 0.07 & 3.634 & 0.465 & 0.006 & 3.573 & 0.011 & 9.018 & 0.027 \\
\hline 11 & 74.80 & 5.002 & -117.10 & 0.07 & 3.697 & 0.481 & 0.006 & 3.665 & 0.011 & 9.202 & 0.041 \\
\hline \multirow[t]{6}{*}{12} & 49.87 & 5.001 & -109.40 & 0.08 & 3.708 & 0.482 & 0.006 & 3.677 & 0.011 & 9.837 & 0.041 \\
\hline & 74.80 & 5.002 & -117.13 & 0.07 & 3.697 & 0.481 & 0.006 & 3.665 & 0.011 & 9.202 & 0.041 \\
\hline & 99.79 & 5.002 & -125.63 & 0.06 & 3.698 & 0.481 & 0.006 & 3.666 & 0.011 & 8.638 & 0.041 \\
\hline & 124.77 & 5.007 & -135.79 & 0.05 & 3.720 & 0.482 & 0.006 & 3.690 & 0.011 & 8.116 & 0.041 \\
\hline & 149.77 & 5.012 & -146.22 & 0.05 & 3.742 & 0.483 & 0.006 & 3.713 & 0.011 & 7.646 & 0.041 \\
\hline & 174.77 & 5.020 & -157.82 & 0.04 & 3.775 & 0.484 & 0.006 & 3.748 & 0.011 & 7.207 & 0.041 \\
\hline \multirow[t]{3}{*}{14} & 0.00 & 5.000 & -100.41 & 0.11 & 3.855 & 0.486 & 0.006 & 3.832 & 0.011 & 11.412 & 0.041 \\
\hline & 24.89 & 5.000 & -103.99 & 0.09 & 3.760 & 0.484 & 0.006 & 3.733 & 0.011 & 10.555 & 0.041 \\
\hline & 49.85 & 5.000 & -109.79 & 0.08 & 3.714 & 0.482 & 0.006 & 3.684 & 0.011 & 9.832 & 0.041 \\
\hline
\end{tabular}

tion (1) which is strictly isocoulombic. Moreover, in this form the equilibria exhibit relatively small heat capacity and volume changes, ${ }^{(34)}$ thus simplifying the fitting equations, as well as the extrapolation to infinite dilution, and the computation of thermodynamic quantities.

Following previous work from this laboratory, ${ }^{(30,31)}$ the quotients for equilibrium (8) were fitted as functions of temperature and ionic strength using the following general equation

$$
\begin{aligned}
\log Q_{1 \mathrm{~b}}= & p_{1}+p_{2} / T+p_{3} \ln T+p_{4} I / T \\
& +p_{5}[1-(1+2 \sqrt{I}) \exp -2 \sqrt{I}] / T-\log \left(a_{\mathrm{w}}\right)
\end{aligned}
$$

where $a_{\mathrm{w}}$ is the activity of water. ${ }^{(35)}$ A weighted regression was performed using the ORGLS general least squares computer program ${ }^{(36)}$ incorporating the data presented in Table III, as well as selected data from Table I. The first three terms in Eq. (12) determine the equilibrium constant $\left(K_{1 \mathrm{~b}}\right)$, whereas the fourth and fifth terms have the same form as terms involving $\beta^{0}$ and $\beta^{1}$, respectively, in the ion interaction model of Pitzer. ${ }^{(37)}$ The values determined for these five parameters are $p_{1}=$ $-19.8878, p_{2}=4113.51, p_{3}=3.30080, p_{4}=12.5746, p_{5}=60.4908$. The isothermal fits of the first dissociation quotient are compared with the experimental and published data in Fig. 1. It is immediately apparent from this figure that the majority of the previous work at elevated ionic strengths deviate considerably from the present fit. Indeed only the value of Cruywagen et al. ${ }^{(13)}$ shows good agreement. This study was carried out in $1 \mathrm{~m} \mathrm{NaCl}$, which may lead to the conclusion that specific 


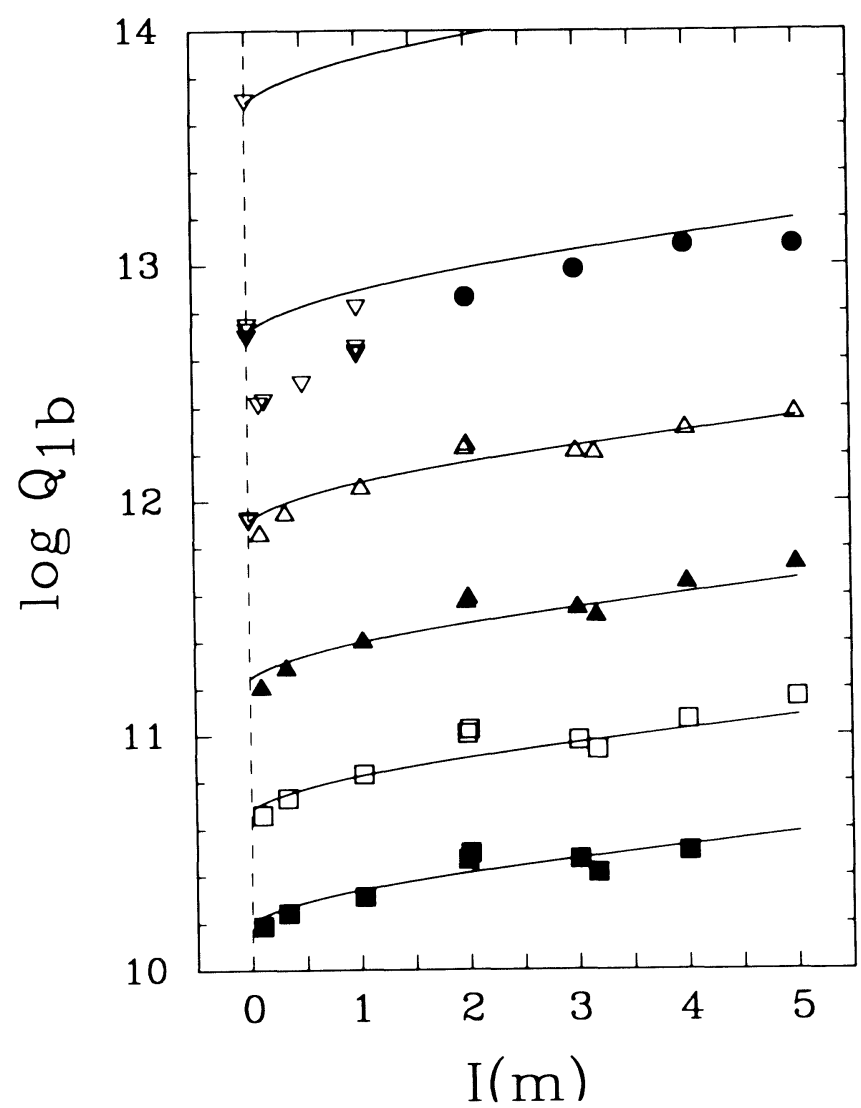

Fig. 1. The relationship between $\log Q_{1 \mathrm{~b}}$ and the ionic strength of the solution at the temperatures: $25^{\circ} \mathrm{C}, \bullet ; 50^{\circ} \mathrm{C}, \Delta ; 75^{\circ} \mathrm{C}$, filled triangle; $100^{\circ} \mathrm{C}, \square$; and $125^{\circ} \mathrm{C}$, filled square. The solid curves result from Eq. (12). The literature values at 0,25 and $50^{\circ} \mathrm{C}$ listed in Table I are represented by $\nabla$.

anion interactions of either $\mathrm{ClO}_{4}^{-(7-12)}$ or $\mathrm{NO}_{3}^{-(14-15)}$ with the oxalate moiety are responsible. However, the corresponding results for $Q_{2 b}$ in Fig. 2 do not show this disparity and hence the differences are probably traceable to the difficulty in determining accurate equilibrium values under conditions (i.e., low temperature and low concentration) where oxalic acid is a relatively strong acid. Note that the results obtained in this study under those conditions were too irreproducible to be included therein. Only the precision of Harned cell $^{(16)}$ and careful glass electrode measurements, ${ }^{(23)}$ which were carried out at high total oxalate concentrations, appear to have minimized these difficulties as exemplified in Fig. 1. 


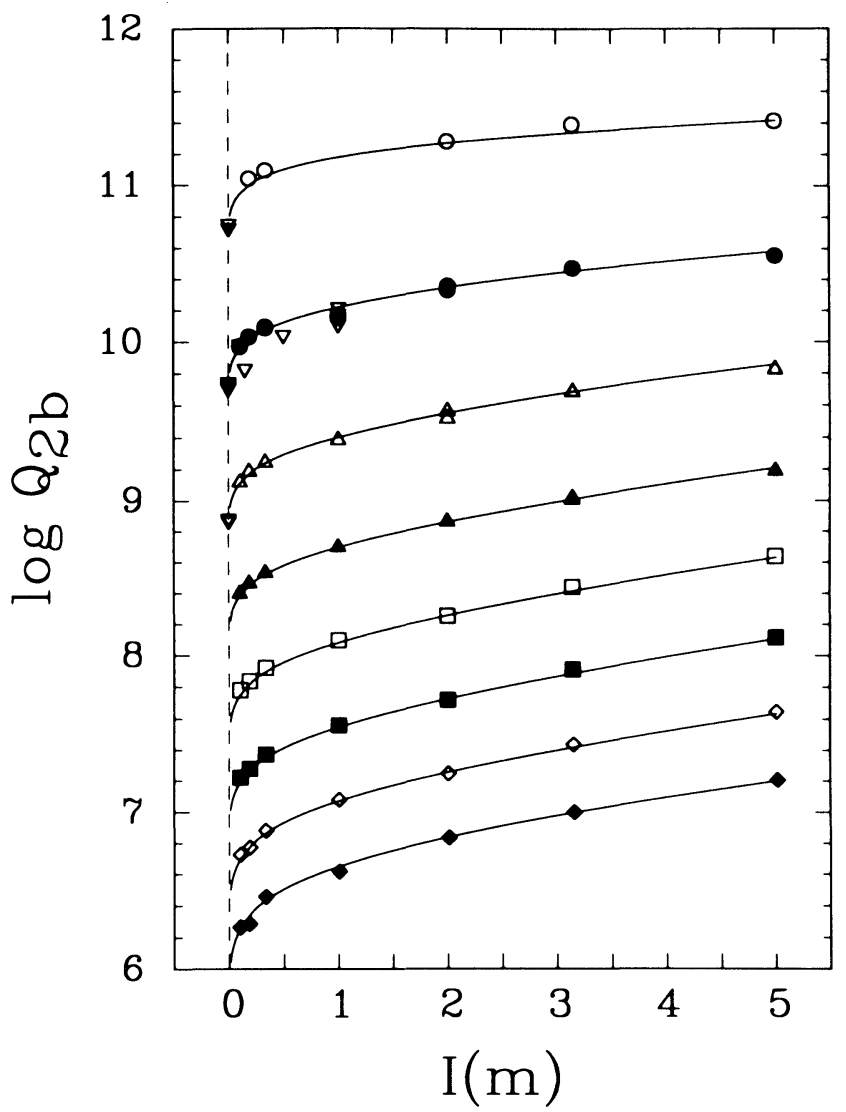

Fig. 2. The relationship between $\log Q_{2 \mathrm{~b}}$ and the ionic strength of the solution at the temperatures: $0^{\circ} \mathrm{C}, \mathrm{o} ; 25^{\circ} \mathrm{C}, \bullet ; 50^{\circ} \mathrm{C}, \Delta ; 75^{\circ} \mathrm{C}$, filled triangle; $100^{\circ} \mathrm{C},[] ; 125^{\circ} \mathrm{C}$, filled square; $150^{\circ} \mathrm{C}, \diamond$; and $175^{\circ} \mathrm{C}$, $\diamond$. The solid curves result from Eq. (14). The literature values at 0,25 and $50^{\circ} \mathrm{C}$ listed in Table I are represented by $\nabla$.

The agreement factor

$$
A F=\left[\left\{\sum W_{\mathrm{i}}\left(Y_{\mathrm{obs}}-Y_{\text {calc }}\right)^{2}\right\} /\left(N-N_{\mathrm{v}}\right)\right]^{1 / 2}
$$

for the fit is 1.58 , where $W$ is the squared reciprocal of the estimated error in any one input datum $\left(Y_{\mathrm{obs}}=\log Q_{\mathrm{lb}}\right.$ or $\left.\Delta H_{1 \mathrm{~b}}\right)$ and $Y_{\text {calc }}$ is derived from Eq. (12), $N$ is the number of $Y_{\mathrm{obs}}$ values, and $N_{\mathrm{v}}$ is the number of independent variables. This $A F$ factor indicates that the actual scatter in the data around the fitting function in Eq. (12) is in effect 1.58 times 

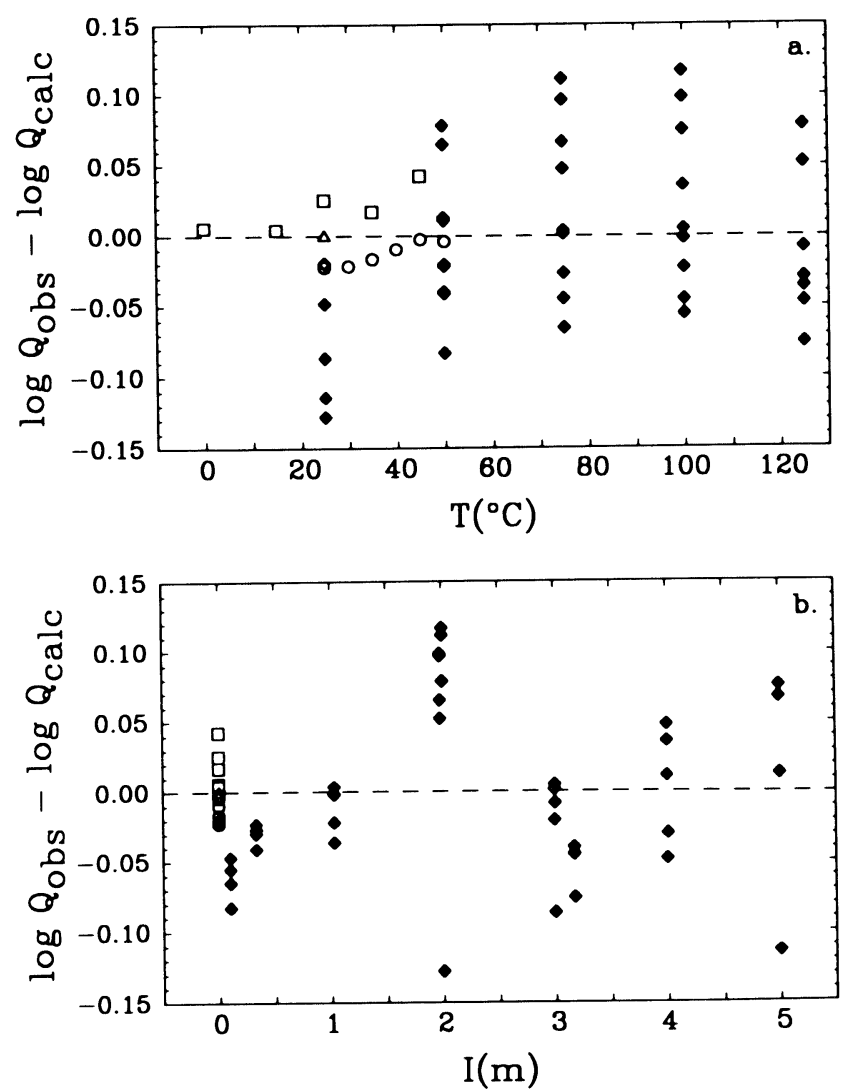

Fig. 3. Deviation plot of the differences between experimental values of $\log Q_{1 \mathrm{~b}}$ and values computed from Eq. (12) as a function of a) temperature; and b) ionic strength. The symbols represent: $\bullet$, this study; ${ }^{[]}$, Ref. 16 ; o, Ref. 23; $\Delta$, Ref. 20; and $\diamond$, Ref. 18.

larger than anticipated based on a statistical summation of 18 possible sources of error in the experimental technique. The average deviation of the $\log Q_{1 \mathrm{~b}}$ values given in Table III is 0.052 as compared with an average deviation of 0.015 for the literature values (Table I) used in the fit. The calorimetrically-determined heat of reaction reported by Christensen et al. ${ }^{(22)}$ agreed to within $175 \mathrm{~J}-\mathrm{mol}^{-1}$ of the calculated value, well within the reported error limits of $310 \mathrm{~J}^{-\mathrm{mol}^{-1}}$. Plots of the residuals of the observed - calculated values of $\log Q_{1 \mathrm{~b}}$ against temperature and ionic strength (Fig. 3) show a random distribution.

Numerical differentiation of Eq. (12) using the ORGLS program yields $\Delta H_{1 \mathrm{~b}}, \Delta S_{1 \mathrm{~b}}$, and $\Delta C_{\mathrm{p}, 1 \mathrm{~b}}$, which along with $\log Q_{1 \mathrm{~b}}$, were converted 
Table V. Summary of Thermodynamic Quantities for the First Acid Dissociation Quotient of Oxalic Acid in Aqueous Sodium Chloride Media at the Saturation Vapor Pressure ${ }^{a}$

\begin{tabular}{|c|c|c|c|c|}
\hline$T\left({ }^{\circ} \mathrm{C}\right)$ & $\log Q_{1 \mathbf{a}}$ & $\Delta H_{1 \mathrm{a}}{ }^{b}$ & $\Delta S_{1 \mathrm{a}}{ }^{c}$ & $\Delta \mathrm{C}_{\mathrm{p}, 1 \mathrm{a}}{ }^{c}$ \\
\hline \multicolumn{5}{|c|}{$I=0.0$} \\
\hline 0 & $-1.252 \pm 0.026$ & $1.1 \pm 1.8$ & $-20 \pm 6$ & $-254 \pm 41$ \\
\hline 25 & $-1.277 \pm 0.010$ & $-4.1 \pm 1.1$ & $-38 \pm 4$ & $-168 \pm 41$ \\
\hline 50 & $-1.358 \pm 0.015$ & $-7.7 \pm 1.0$ & $-50 \pm 3$ & $-123 \pm 41$ \\
\hline 75 & $-1.463 \pm 0.025$ & $-10.5 \pm 1.8$ & $-58 \pm 5$ & $-106 \pm 41$ \\
\hline 100 & $-1.581 \pm 0.044$ & $-13.1 \pm 2.7$ & $-65 \pm 8$ & $-111 \pm 41$ \\
\hline 125 & $-1.709 \pm 0.069$ & $-16.2 \pm 3.7$ & $-73 \pm 10$ & $-133 \pm 41$ \\
\hline \multicolumn{5}{|c|}{$I=0.1$} \\
\hline 0 & $-1.013 \pm 0.035$ & $1.4 \pm 1.8$ & $-14 \pm 6$ & $-247 \pm 41$ \\
\hline 25 & $-1.032 \pm 0.025$ & $-3.5 \pm 1.1$ & $-32 \pm 4$ & $-156 \pm 41$ \\
\hline 50 & $-1.102 \pm 0.021$ & $-6.7 \pm 1.1$ & $-42 \pm 3$ & $-105 \pm 41$ \\
\hline 75 & $-1.193 \pm 0.021$ & $-9.0 \pm 1.8$ & $-49 \pm 5$ & $-85 \pm 41$ \\
\hline 100 & $-1.294 \pm 0.035$ & $-11.1 \pm 2.8$ & $-55 \pm 8$ & $-86 \pm 41$ \\
\hline 125 & $-1.401 \pm 0.060$ & $-13.5 \pm 3.7$ & $-61 \pm 10$ & $-104 \pm 41$ \\
\hline \multicolumn{5}{|c|}{$I=0.5$} \\
\hline 0 & $-0.854 \pm 0.071$ & $1.3 \pm 1.8$ & $-12 \pm 6$ & $-239 \pm 41$ \\
\hline 25 & $-0.873 \pm 0.063$ & $-3.4 \pm 1.2$ & $-28 \pm 4$ & $-142 \pm 41$ \\
\hline 50 & $-0.939 \pm 0.054$ & $-6.2 \pm 1.3$ & $-37 \pm 3$ & $-87 \pm 41$ \\
\hline 75 & $-1.022 \pm 0.044$ & $-8.0 \pm 2.0$ & $-42 \pm 5$ & $-62 \pm 41$ \\
\hline 100 & $-1.109 \pm 0.040$ & $-9.4 \pm 2.9$ & $-47 \pm 8$ & $-59 \pm 41$ \\
\hline 125 & $-1.198 \pm 0.054$ & $-11.1 \pm 3.9$ & $-51 \pm 10$ & $-72 \pm 41$ \\
\hline \multicolumn{5}{|c|}{$I=1.0$} \\
\hline 0 & $-0.803 \pm 0.087$ & $1.0 \pm 1.8$ & $-12 \pm 6$ & $-231 \pm 41$ \\
\hline 25 & $-0.826 \pm 0.078$ & $-3.4 \pm 1.2$ & $-27 \pm 4$ & $-130 \pm 41$ \\
\hline 50 & $-0.891 \pm 0.068$ & $-5.9 \pm 1.3$ & $-35 \pm 3$ & $-72 \pm 41$ \\
\hline 75 & $-0.967 \pm 0.055$ & $-7.3 \pm 2.1$ & $-39 \pm 5$ & $-43 \pm 41$ \\
\hline 100 & $-1.045 \pm 0.047$ & $-8.2 \pm 3.0$ & $-42 \pm 8$ & $-38 \pm 41$ \\
\hline 125 & $-1.121 \pm 0.054$ & $-9.3 \pm 3.9$ & $-45 \pm 10$ & $-47 \pm 41$ \\
\hline \multicolumn{5}{|c|}{$I=3.0$} \\
\hline 0 & $-0.871 \pm 0.070$ & $-0.1 \pm 1.8$ & $-17 \pm 6$ & $-202 \pm 41$ \\
\hline 25 & $-0.935 \pm 0.061$ & $-3.7 \pm 1.2$ & $-30 \pm 4$ & $-92 \pm 41$ \\
\hline 50 & $-0.966 \pm 0.052$ & $-5.1 \pm 1.3$ & $-34 \pm 3$ & $-25 \pm 41$ \\
\hline 75 & $-1.027 \pm 0.040$ & $-5.2 \pm 2.0$ & $-35 \pm 5$ & $12 \pm 41$ \\
\hline 100 & $-1.076 \pm 0.036$ & $-4.6 \pm 2.9$ & $-33 \pm 8$ & $27 \pm 41$ \\
\hline 125 & $-1.112 \pm 0.050$ & $-3.9 \pm 3.9$ & $-31 \pm 10$ & $28 \pm 41$ \\
\hline
\end{tabular}


Table V. Continued

\begin{tabular}{rccrr}
\hline$T\left({ }^{\circ} \mathrm{C}\right)$ & $\log Q_{1 \mathrm{a}}$ & $\Delta H_{1 \mathrm{a}}{ }^{b}$ & $\Delta S_{1 \mathrm{a}}{ }^{c}$ & $\Delta \mathrm{C}_{\mathrm{p}, 1 \mathrm{a}}{ }^{c}$ \\
\hline & & $I=5.0$ & \\
0 & $-1.052 \pm 0.097$ & $-1.2 \pm 1.9$ & $-24 \pm 6$ & $-175 \pm 41$ \\
25 & $-1.096 \pm 0.086$ & $-4.0 \pm 1.2$ & $-34 \pm 4$ & $-58 \pm 41$ \\
50 & $-1.154 \pm 0.078$ & $-4.4 \pm 1.2$ & $-36 \pm 3$ & $18 \pm 41$ \\
75 & $-1.200 \pm 0.074$ & $-3.4 \pm 1.9$ & $-33 \pm 5$ & $62 \pm 41$ \\
100 & $-1.223 \pm 0.076$ & $-1.5 \pm 2.8$ & $-27 \pm 8$ & $85 \pm 41$ \\
125 & $-1.224 \pm 0.088$ & $0.8 \pm 3.7$ & $-22 \pm 10$ & $94 \pm 41$ \\
\hline
\end{tabular}

${ }^{a}$ Errors listed represent three times the standard deviation. ${ }^{b}$ Units: $\mathrm{kJ}^{-\mathrm{mol}^{-1}}{ }^{c}{ }^{c}$ Units: $\mathrm{J}-\mathrm{K}^{-1}-\mathrm{mol}^{-1}$.

to the corresponding values for the dissociation reaction (reaction 1) by the incorporation of the appropriate thermodynamic quantities for the dissociation of water. ${ }^{(33)}$ These quantities are presented in Table V . The values for $\Delta C_{\mathrm{p}, 1 \mathrm{a}}$ are slightly greater than those for the dissociation of water, ${ }^{(33)}$ by a margin outside the experimental errors and show the same trends with temperature and ionic strength. These trends are largely due to the fact that the heat capacity of water is involved directly in the anionic treatment.

A similar regression of the $\log Q_{2 \mathrm{~b}}$ values was accomplished using the general equation

$$
\begin{aligned}
\log Q_{2 \mathrm{~b}}=2 & A_{\phi}\{\sqrt{I} /(1+1.2 \sqrt{I})+(2 / 1.2) \ln (1+1.2 \sqrt{I})\} /(\ln 10) \\
& -\log \left(a_{\mathrm{w}}\right)+p_{1}+p_{2} / T+p_{3} T+p_{4} T^{2} I+p_{5} I / T \\
& +p_{6} I+p_{7} T\{1-(1+2 \sqrt{I}) \exp -2 \sqrt{I}\}
\end{aligned}
$$

where the first term corresponds to the Debye-Hückel expression, ${ }^{(38)}$ the $p_{1}-p_{3}$ terms pertain to the equilibrium constant, $K_{1 \mathrm{~b}}$, the $p_{4}-p_{6}$ terms have the form of $\beta^{0}$, and the $p_{7}$ term simulates the form of $\beta^{1}$, in the Pitzer treatment. ${ }^{(37)}$ The data are best represented by the following parameters $p_{1}=0.315529, p_{2}=3037.87, p_{3}=-2.63797 \times 10^{-3}, p_{4}=$ $-1.44644 \times 10^{-6}, p_{5}=-159.261, p_{6}=0.668164$, and $p_{7}=-6.67308 \times 10^{-4}$.

The agreement factor from this fit is 1.45 . A deviation plot of the results for $\log Q_{2 \mathrm{~b}}$ given in Table IV from those calculated from Eq. (14) is presented in Fig. 4. The average deviation of these $\log Q_{2 \mathrm{~b}}$ results is 0.018 , compared with 0.010 for data obtained from the literature. Although the residuals of the linear regression exhibit no systematic rela- 

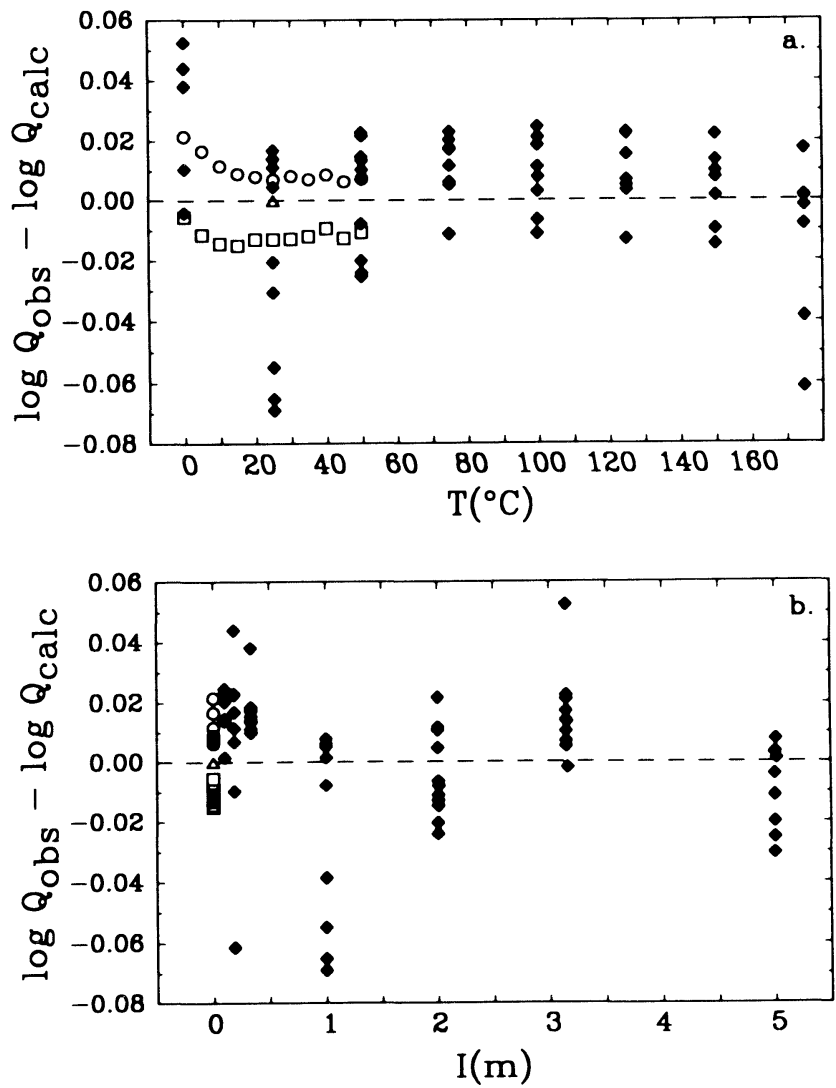

Fig. 4. Deviation plot of the differences between experimental values of $\log Q_{2 b}$ and values computed from Eq. (14) as a function of a) temperature; and b) ionic strength. The symbols represent: $\bullet$, this study; $[$, Ref. 19; o, Ref. 21; and $\Delta$, Ref. 20.

tion to ionic strength, the deviations between observed and calculated values of $\log Q_{2 \mathrm{~b}}$ decrease with increasing temperature. The data at $150^{\circ}$ and $175^{\circ} \mathrm{C}$ are less precise than those obtained at temperatures between $75^{\circ}$ and $125^{\circ} \mathrm{C}$ as a consequence of the correction for drift at the higher temperatures. Nevertheless, the deviations observed at $150^{\circ}$ are small relative to those observed at temperatures below $50^{\circ} \mathrm{C}$ (Fig. 4).

The data obtained for the determination of $\log Q_{2 \mathrm{~b}}$ are more precise than those obtained for $\log Q_{1 \mathrm{~b}}$. This difference reflects the low $\bar{n}$ values obtained in runs to determine the first dissociation quotient. The greatest precision and accuracy will be obtained when $\bar{n}=1.5$ for 
the first dissociation constant and when $\bar{n}=0.5$ for determinations of the second dissociation constant. The values of $\bar{n}$ obtained for experiments 1-16 are in good agreement with the desired value (Table IV). Values of $\bar{n}$ obtained in experiments 17-31 are considerably less than 1.5 (Table III).

Numerical differentiation of Eq. (14) using the ORGLS program yields $\Delta H_{2 \mathrm{~b}}, \Delta S_{2 \mathrm{~b}}$, and $\Delta C_{\mathrm{p}, 2 \mathrm{~b}}$, which along with $\log Q_{2 \mathrm{~b}}$, were converted to the corresponding values for the dissociation reaction (reaction 2 ) by incorporation of the appropriate thermodynamic quantities for the dissociation of water. ${ }^{(33)}$ The equilibrium quotients for the dissociation reaction and the relevant thermodynamic data were derived as described above and are presented in Table VI. The agreement of $\Delta H_{2 \mathrm{a}}^{\circ}$ at $25^{\circ} \mathrm{C}$ with the calorimetric value ${ }^{(22)}$ used in the fit is considered acceptable. The general behavior of $\Delta H, \Delta S$ and $\Delta C_{\mathrm{p}}$ for both reactions are typical of all acid and base equilibria studied to high temperatures to date. ${ }^{(24)}$ In other words, the acids become more rapidly weak as the critical point of water is approached due to the entropy change which dominates the opposing effect of the enthalpy.

The equations relating the dissociation constants at infinite dilution to temperature are

$$
\begin{aligned}
\log K_{1 \mathrm{a}}= & -626.410+35399.5 / T+98.2742 \ln T \\
& -0.097611 T-2.17087 \times 10^{6} / T^{2}
\end{aligned}
$$

and

$$
\begin{aligned}
\log K_{2 \mathrm{a}}= & -606.206+34323.9 / T+94.9734 \ln T \\
& -0.100249 T-2.17087 \times 10^{6} / T^{2}
\end{aligned}
$$

These relations can be compared directly with those reported by Nikolaeva and Antipina ${ }^{(17)}$ (Figs. 5 and 6). Although agreement for the second dissociation constant is quite good, there are substantial deviations between the present results for the first dissociation constant and published equation. The latter equation relating $\log K_{1 \mathrm{a}}$ and $T$ can be rejected for three reasons. 1) All measurements reported by Nikolaeva and Antipina were made in relatively dilute solutions $(I<0.13)$, over a narrow range of ionic strength (e.g., 0.055 to 0.126 at $25^{\circ} \mathrm{C}$ ). Small errors in the dissociation quotient will then be magnified during extrapolation to infinite dilution. 2) The dissociation quotient was considered to be independent of ionic strength at $25^{\circ} \mathrm{C}$. 3) Data at other temperatures were extrapolated to infinite dilution as a linear function of $\sqrt{I}$. Also, Eq. (15) is seen to be in quantitative agreement with the very 
Table VI. Summary of Thermodynamic Quantities for the Second Dissociation Quotient of Oxalic Acid in Aqueous Sodium Chloride Media at the Saturation Vapor Pressure ${ }^{a}$

\begin{tabular}{|c|c|c|c|c|}
\hline$T\left({ }^{\circ} \mathrm{C}\right)$ & $\log Q_{2 \mathbf{a}}$ & $\Delta H_{2 \mathrm{a}}$ & $\Delta S_{2 \mathrm{a}}$ & $\Delta \mathrm{C}_{\mathrm{p}, 2 \mathrm{a}}$ \\
\hline \multicolumn{5}{|c|}{$I=0.0$} \\
\hline 0 & $-4.224 \pm 0.010$ & $0.6 \pm 0.7$ & $-79 \pm 2$ & $-344 \pm 11$ \\
\hline 25 & $-4.275 \pm 0.006$ & $-6.8 \pm 0.5$ & $-105 \pm 2$ & $-261 \pm 12$ \\
\hline 50 & $-4.408 \pm 0.008$ & $-12.8 \pm 0.4$ & $-124 \pm 1$ & $-218 \pm 13$ \\
\hline 75 & $-4.586 \pm 0.011$ & $-18.0 \pm 0.5$ & $-139 \pm 2$ & $-204 \pm 14$ \\
\hline 100 & $-4.792 \pm 0.016$ & $-23.2 \pm 0.8$ & $-154 \pm 2$ & $-212 \pm 15$ \\
\hline 125 & $-5.019 \pm 0.022$ & $-28.7 \pm 1.2$ & $-168 \pm 3$ & $-237 \pm 16$ \\
\hline 150 & $-5.264 \pm 0.030$ & $-35.1 \pm 1.5$ & $-184 \pm 4$ & $-275 \pm 17$ \\
\hline 175 & $-5.529 \pm 0.041$ & $-42.6 \pm 2.0$ & $-201 \pm 5$ & $-326 \pm 18$ \\
\hline \multicolumn{5}{|c|}{$I=0.1$} \\
\hline 0 & $-3.796 \pm 0.012$ & $1.2 \pm 0.7$ & $-68 \pm 2$ & $-340 \pm 11$ \\
\hline 25 & $-3.826 \pm 0.008$ & $-6.1 \pm 0.5$ & $-94 \pm 2$ & $-252 \pm 11$ \\
\hline 50 & $-3.934 \pm 0.009$ & $-11.7 \pm 0.4$ & $-112 \pm 1$ & $-204 \pm 12$ \\
\hline 75 & $-4.082 \pm 0.010$ & $-16.5 \pm 0.5$ & $-126 \pm 1$ & $-187 \pm 13$ \\
\hline 100 & $-4.253 \pm 0.012$ & $-21.2 \pm 0.7$ & $-138 \pm 2$ & $-191 \pm 14$ \\
\hline 125 & $-4.438 \pm 0.017$ & $-26.2 \pm 1.1$ & $-151 \pm 3$ & $-211 \pm 15$ \\
\hline 150 & $-4.637 \pm 0.025$ & $-31.8 \pm 1.5$ & $-164 \pm 4$ & $-242 \pm 16$ \\
\hline 175 & $-4.847 \pm 0.035$ & $-38.4 \pm 1.9$ & $-178 \pm 5$ & $-280 \pm 17$ \\
\hline \multicolumn{5}{|c|}{$I=0.5$} \\
\hline 0 & $-3.568 \pm 0.019$ & $2.0 \pm 0.7$ & $-61 \pm 3$ & $-338 \pm 10$ \\
\hline 25 & $-3.577 \pm 0.017$ & $-5.2 \pm 0.5$ & $-86 \pm 2$ & $-246 \pm 11$ \\
\hline 50 & $-3.662 \pm 0.018$ & $-10.6 \pm 0.4$ & $-103 \pm 1$ & $-195 \pm 12$ \\
\hline 75 & $-3.784 \pm 0.017$ & $-15.2 \pm 0.4$ & $-116 \pm 1$ & $-174 \pm 13$ \\
\hline 100 & $-3.924 \pm 0.017$ & $-19.5 \pm 0.6$ & $-127 \pm 2$ & $-175 \pm 14$ \\
\hline 125 & $-4.076 \pm 0.018$ & $-24.1 \pm 0.9$ & $-138 \pm 2$ & $-193 \pm 15$ \\
\hline 150 & $-4.235 \pm 0.021$ & $-29.2 \pm 1.3$ & $-150 \pm 3$ & $-219 \pm 16$ \\
\hline 175 & $-4.401 \pm 0.028$ & $-35.0 \pm 1.7$ & $-162 \pm 4$ & $-246 \pm 17$ \\
\hline \multicolumn{5}{|c|}{$I=1.0$} \\
\hline 0 & $-3.506 \pm 0.026$ & $2.8 \pm 1.0$ & $-57 \pm 3$ & $-338 \pm 11$ \\
\hline 25 & $-3.497 \pm 0.021$ & $-4.3 \pm 0.7$ & $-82 \pm 2$ & $-243 \pm 12$ \\
\hline 50 & $-3.564 \pm 0.021$ & $-9.7 \pm 0.5$ & $-98 \pm 2$ & $-190 \pm 13$ \\
\hline 75 & $-3.666 \pm 0.022$ & $-14.1 \pm 0.4$ & $-111 \pm 1$ & $-167 \pm 15$ \\
\hline 100 & $-3.786 \pm 0.022$ & $-18.2 \pm 0.6$ & $-121 \pm 2$ & $-167 \pm 16$ \\
\hline 125 & $-3.915 \pm 0.021$ & $-22.6 \pm 0.9$ & $-132 \pm 2$ & $-183 \pm 18$ \\
\hline 150 & $-4.050 \pm 0.024$ & $-27.4 \pm 1.4$ & $-142 \pm 4$ & $-206 \pm 20$ \\
\hline 175 & $-4.189 \pm 0.029$ & $-32.9 \pm 1.9$ & $-154 \pm 5$ & $-229 \pm 22$ \\
\hline
\end{tabular}


Table VI. Continued

\begin{tabular}{rcccc}
\hline$T\left({ }^{\circ} \mathrm{C}\right)$ & $\log Q_{2 \mathrm{a}}$ & $\Delta H_{2 \mathrm{a}}$ & $\Delta S_{2 \mathrm{a}}$ & $\Delta \mathrm{C}_{\mathrm{p}, 2 \mathrm{a}}$ \\
\hline & & & \\
0 & $-3.600 \pm 0.058$ & $6.1 \pm 2.5$ & $-47 \pm 8$ & $-336 \pm 22$ \\
25 & $-3.560 \pm 0.032$ & $-0.9 \pm 2.0$ & $-71 \pm 6$ & $-236 \pm 26$ \\
50 & $-3.538 \pm 0.028$ & $-6.1 \pm 1.4$ & $-87 \pm 4$ & $-180 \pm 31$ \\
75 & $-3.585 \pm 0.030$ & $-10.2 \pm 1.0$ & $-98 \pm 3$ & $-154 \pm 36$ \\
100 & $-3.647 \pm 0.030$ & $-14.0 \pm 1.2$ & $-107 \pm 3$ & $-151 \pm 41$ \\
125 & $-3.717 \pm 0.029$ & $-17.9 \pm 2.1$ & $-116 \pm 5$ & $-163 \pm 47$ \\
150 & $-3.792 \pm 0.036$ & $-22.2 \pm 3.3$ & $-125 \pm 8$ & $-181 \pm 53$ \\
175 & $-3.866 \pm 0.055$ & $-26.9 \pm 4.6$ & $-134 \pm 11$ & $-195 \pm 59$ \\
& & $I=5.0$ & & \\
0 & $-3.838 \pm 0.101$ & $9.4 \pm 4.2$ & $-39 \pm 14$ & $-334 \pm 37$ \\
25 & $-3.709 \pm 0.061$ & $2.4 \pm 3.3$ & $-63 \pm 11$ & $-232 \pm 44$ \\
50 & $-3.665 \pm 0.054$ & $-2.6 \pm 2.3$ & $-78 \pm 7$ & $-173 \pm 52$ \\
75 & $-3.661 \pm 0.058$ & $-6.5 \pm 1.7$ & $-89 \pm 5$ & $-145 \pm 60$ \\
100 & $-3.676 \pm 0.057$ & $-10.0 \pm 2.1$ & $-97 \pm 6$ & $-140 \pm 69$ \\
125 & $-3.699 \pm 0.056$ & $-13.6 \pm 3.6$ & $-105 \pm 9$ & $-150 \pm 79$ \\
150 & $-3.727 \pm 0.067$ & $-17.6 \pm 5.6$ & $-113 \pm 14$ & $-166 \pm 90$ \\
175 & $-3.754 \pm 0.098$ & $-21.9 \pm 7.9$ & $-121 \pm 19$ & $-175 \pm 101$ \\
\hline
\end{tabular}

${ }^{a}$ See footnotes of Table V.

precise low temperature measurements of McAuley and Nancollas ${ }^{(16)}$ and Kurz and Farrar. ${ }^{(23)}$

\section{Conclusions}

The results of this study are shown to be in quantitative agreement with a number of previous studies at low temperatures and ionic strengths. Equations $(12,14,15,16)$ provide an accurate model for the speciation of oxalic acid in $\mathrm{NaCl}$ brines to high temperatures and salinities, as well as in pure water. These results can now be used in the determination of the $\mathrm{pH}$ of solutions encountered in industrial settings such as power plants and waste treatment streams, and in natural hydrothermal fluids. Moreover, experimental studies of the type carried out recently in this laboratory on the formation of ferrous acetate complexes $^{(39)}$ are now possible for metal-oxalate complexes in high temperature chloride brines. Such information is critical to an understanding of the importance and effects of dissolved carboxylic acids in groundwater/soil interaction, as well as in the evolution of permeability, acidity and metal transport in sedimentary basins and oilfield settings. 


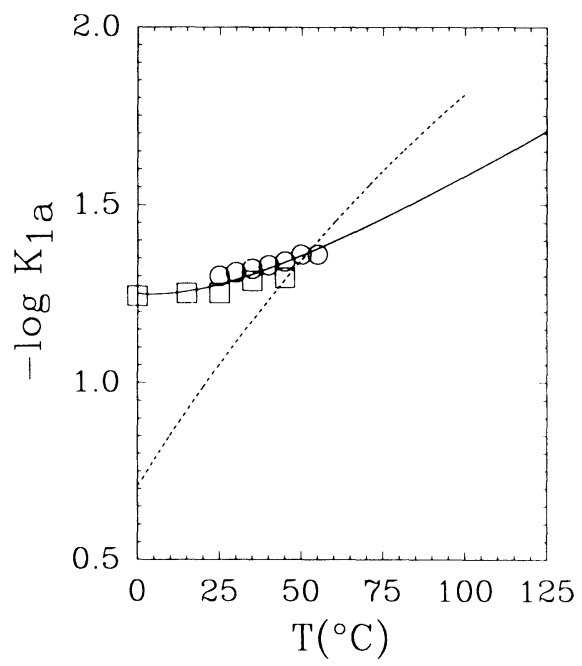

Fig. 5. The $\log K_{1 \mathrm{a}}$ is shown as a function of temperature based on Eq. (15) (solid line); the results in Table I of Ref. 16, [], and Ref. 23, o; and the equation reported by Ref. 17 (dashed line).

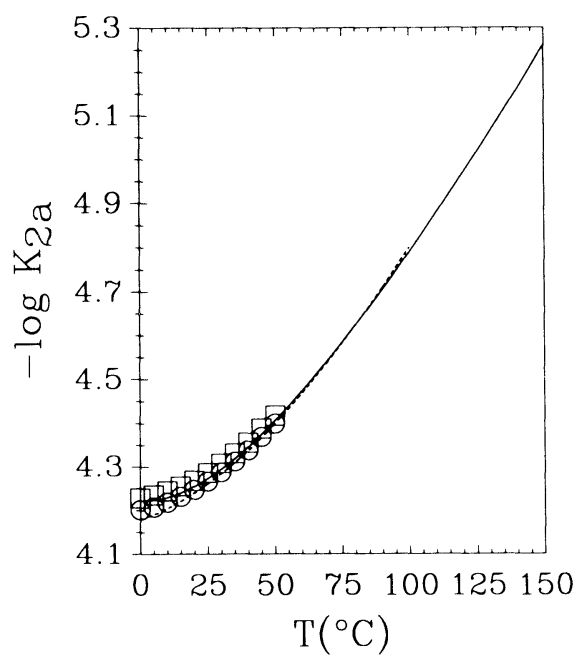

Fig. 6. The $\log K_{2 \mathrm{a}}$ is shown as a function of temperature based on Eq. (16) (solid line); the equation reported by Ref. 17 (dashed line); and the results in Table I of Ref. 19, $]$, and Ref. 21, o. 


\section{Acknowledgment}

We thank Vitaly Zlotnik for translating Ref. 17. This research was carried out at Oak Ridge National Laboratory while RMK was a summer faculty participant with Oak Ridge Associated Universities. Financial support for this project was provided by the Office of Basic Energy Sciences of the U. S. Department of Energy under contract DEAC05-84OR21400 with Martin Marietta Energy Systems, Inc. RMK would also like to thank the Research Council of the University of Nebraska for their financial support.

\section{References}

1. A. P. Murray and L. F. Becker, Jr., Water Chem. Nucl. React. Sys. 4, 275 (1986).

2. J. Akatsu, Sep. Sci. and Tech. 17, 1433 (1983).

3. I. Sekine and C. Okano, Corrosion 45, 924 (1988).

4. W. C. Graustein, K. Cromack, Jr., and P. Sollins, Science 198, 1252 (1977).

5. P. C. Bennett, M. E. Melcer, D. I. Siegel, and J. P. Hassett, Geochim. Cosmochim. Acta 52, 1521 (1988).

6. R. C. Surdam and L. J. Crossey, Phil. Trans. R. Soc. Lond. A315, 135 (1985).

7. A. McAuley and G. H. Nancollas, Trans. Faraday Soc. 56, 1165 (1960).

8. H. J. de Bruin, D. Kairaitis, and R. K. Temple, Report AAEC/E-72, 1961.

9. R. F. Bauer and W. M. Smith, Can. J. Chem. 43, 2755 (1965).

10. E. Bottari and L. Ciavatta, Gazz. Chim. Ital. 95, 908 (1965).

11. E. G. Moorhead and N. Sutin, N. Inorg. Chem. 5, 1866 (1966).

12. I. Grenthe, G. Gårdhammer, and E. Rundcrantz, Acta Chem. Scand. 23, 93 (1969).

13. R. E. Mesmer, D. A. Palmer, and J. M. Simonson, in Activity Coefficients in Electrolyte Solutions, K. S. Pitzer, ed., (CRC Press, 1991), Chap. 8.

14. K. S. Rajan and A. E. Martell, J. Inorg. Nucl. Chem. 29, 523 (1967).

15. H. K. Lin, Z. Gu, and Y. Chen, Gazz. Chim. Ital. 117, 23 (1987).

16. A. McAuley and G. H. Nancollas, J. Chem. Soc. A 1961, 2215.

17. N. M. Nikolaeva and V. A. Antipina, Akademikita Nauk SSSR 6, 13 (1972).

18. H. N. Parton and R. C. Gibbons, Trans. Faraday Soc. 35, 542 (1939).

19. H. S. Harned and L. D. Fallon, J. Am. Chem. Soc. 61, 3111 (1939).

20. L. S. Darken, J. Am. Chem. Soc. 63, 1007 (1941).

21. G. D. Pinching and R. G. Bates, J. Res. Nat. Bur. Stand. 40, 405 (1948).

22. J. J. Christensen, R. M. Izatt, and L. D. Hansen, J. Am. Chem. Soc. 89, 213 (1967).

23. J. L. Kurz and J. M. Farrar, J. Am. Chem. Soc. 91, 6057 (1969).

24. J. J. Cruywagen, J. B. Heyns, and R. F. van der Water, J. Chem. Soc. Dalton Trans. 1857 (1986).

25. R. E. Mesmer, C. F. Baes, Jr., and F. H. Sweeton, J. Phys. Chem. 74, 1937 (1970).

26. D. J. Wesolowski, S. E. Drummond, R. E. Mesmer, and H. Ohmoto, Inorg. Chem. 23, 1120 (1984).

27. L. J. Crossey, Geochim. Cosmochim. Acta 55, 1515 (1991).

28. C. F. Baes, Jr. and R. E. Mesmer, The Hydrolysis of Cations (Wiley, New York, 
1976).

29. A. S. Quist and W. L. Marshall, J. Phys. Chem. 69, 2984 (1965).

30. D. A. Palmer, D. Wesolowski, and R. E. Mesmer, J. Solution Chem. 16, 443 (1987).

31. D. J. Wesolowski and D. A. Palmer, J. Solution Chem. 18, 545 (1989).

32. B. F. Hitch and R. E. Mesmer, J. Solution Chem. 5, 667 (1976).

33. R. H. Busey and R. E. Mesmer, J. Chem. Eng. Data 23, 175 (1978).

34. W. T. Lindsay, Proc. Int. Water Conf. Eng. Soc., W. Pa. 41, 284 (1980).

35. R. H. Busey and R. E. Mesmer, J. Solution Chem. 5, 147 (1976).

36. W. R. Busing and H. A. Levy, Oak Ridge Natl. Lab. Rep., ORNL-TM (U.S.) ORNL-TM-271 (1963).

37. K. S. Pitzer, J. Phys. Chem. 77, 268 (1973).

38. A. G. Dickson, D. J. Wesolowski, D. A. Palmer, and R. E. Mesmer, J. Phys. Chem 94, 7978 (1990).

39. D. A. Palmer and S. E. Drummond, J. Phys. Chem. 92, 6795 (1988). 